\title{
Data Dependence Results for Multistep and CR Iterative Schemes in the Class of Contractive-Like Operators
}

\author{
Vatan Karakaya, ${ }^{1}$ Faik Gürsoy, ${ }^{2}$ Kadri Doğan, ${ }^{1}$ and Müzeyyen Ertürk ${ }^{2}$ \\ ${ }^{1}$ Department of Mathematical Engineering, Yildiz Technical University, Davutpasa Campus, Esenler, 34210 Istanbul, Turkey \\ ${ }^{2}$ Department of Mathematics, Yildiz Technical University, Davutpasa Campus, Esenler, 34220 Istanbul, Turkey
}

Correspondence should be addressed to Vatan Karakaya; vkkaya@yildiz.edu.tr

Received 17 February 2013; Accepted 10 July 2013

Academic Editor: Adem Kilicman

Copyright (c) 2013 Vatan Karakaya et al. This is an open access article distributed under the Creative Commons Attribution License, which permits unrestricted use, distribution, and reproduction in any medium, provided the original work is properly cited.

We intend to establish some results on the data dependence of fixed points of certain contractive-like operators for the multistep and CR iterative processes in a Banach space setting. One of our results generalizes the corresponding results of Soltuz and Grosan (2008) and Chugh and Kumar (2011).

\section{Introduction}

Throughout this paper, $\mathbb{N}$ denotes the set of all nonnegative integers. Let $X$ be a Banach space, $E \subset X$ a nonempty closed, convex subset of $X$, and $T$ a self-map on $E$. Suppose that $F_{T}:=$ $\{p \in X: p=T p\}$ is the set of all fixed points of $T$. Iterative schemes abound in the literature of fixed point theory for which the fixed points of operators have been approximated over the years by many authors.

It is well known that the Picard iteration procedure [1] is defined by

$$
\begin{gathered}
x_{0} \in E, \\
x_{n+1}=T x_{n}, \quad n \in \mathbb{N} .
\end{gathered}
$$

Let $\left\{\alpha_{n}\right\}_{n=0}^{\infty},\left\{\beta_{n}\right\}_{n=0}^{\infty},\left\{\gamma_{n}\right\}_{n=0}^{\infty}$ and $\left\{\beta_{n}^{i}\right\}_{n=0}^{\infty}, i=\overline{1, k-2}, k \geq 2$ be the real sequences in $[0,1)$ satisfying certain conditions.

The Mann iterative scheme [2] is defined by

$$
\begin{gathered}
x_{0} \in E, \\
x_{n+1}=\left(1-\alpha_{n}\right) x_{n}+\alpha_{n} T x_{n}, \quad n \in \mathbb{N} .
\end{gathered}
$$

If $\alpha_{n}=\lambda$ (constant) in (2), then the resulting iteration will be called Krasnoselkij iteration procedure [3].
A sequence $\left\{x_{n}\right\}_{n=0}^{\infty}$, defined by

$$
\begin{gathered}
x_{0} \in E, \\
x_{n+1}=\left(1-\alpha_{n}\right) x_{n}+\alpha_{n} T y_{n}, \\
y_{n}=\left(1-\beta_{n}\right) x_{n}+\beta_{n} T x_{n}, \quad n \in \mathbb{N},
\end{gathered}
$$

is commonly known as the Ishikawa iterative method [4].

The Noor iterative procedure [5] is defined by

$$
\begin{gathered}
x_{0} \in E, \\
x_{n+1}=\left(1-\alpha_{n}\right) x_{n}+\alpha_{n} T y_{n}, \\
y_{n}=\left(1-\beta_{n}\right) x_{n}+\beta_{n} T z_{n}, \\
z_{n}=\left(1-\gamma_{n}\right) x_{n}+\gamma_{n} T x_{n}, \quad n \in \mathbb{N} .
\end{gathered}
$$

In 2004, Rhoades and Soltuz [6] introduced a multistep iterative process as follows:

$$
\begin{gathered}
x_{0} \in E, \\
x_{n+1}=\left(1-\alpha_{n}\right) x_{n}+\alpha_{n} T y_{n}^{1}, \\
y_{n}^{i}=\left(1-\beta_{n}^{i}\right) x_{n}+\beta_{n}^{i} T y_{n}^{i+1}, \\
y_{n}^{k-1}=\left(1-\beta_{n}^{k-1}\right) x_{n}+\beta_{n}^{k-1} T x_{n}, \quad n \in \mathbb{N} .
\end{gathered}
$$


The iteration processes (2), (3), and (4) can be viewed as the special cases of the iteration procedure (5).

Recently, Chugh et al. introduced a CR iterative scheme in [7] as follows:

$$
\begin{gathered}
x_{0} \in E, \\
x_{n+1}=\left(1-\alpha_{n}\right) y_{n}+\alpha_{n} T y_{n}, \\
y_{n}=\left(1-\beta_{n}\right) T x_{n}+\beta_{n} T z_{n}, \\
z_{n}=\left(1-\gamma_{n}\right) x_{n}+\gamma_{n} T x_{n}, \quad n \in \mathbb{N} .
\end{gathered}
$$

Now we mention some important contractive type operators.

Any mapping $T$ is called a Kannan mapping, see [8], if there exists $b \in(0,1 / 2)$ such that, for all $x, y \in X$,

$$
\|T x-T y\| \leq b(\|x-T x\|+\|y-T y\|) .
$$

Similar mapping is called a Chatterjea mapping, see [9], if there exists $c \in(0,1 / 2)$ such that, for all $x, y \in X$,

$$
\|T x-T y\| \leq c(\|x-T y\|+\|y-T x\|) .
$$

In [10] Zamfirescu collected these classes of operators and proved an important result which states that an operator $T$ : $X \rightarrow X$ satisfies condition $\mathrm{Z}$ (Zamfirescu condition) if and only if there exist the real numbers $a, b$, and $c$ satisfying $0<$ $a<1,0<b$, and $c<1 / 2$ such that, for each pair $x, y \in X$, at least one of the following conditions is true:

$$
\begin{aligned}
& \left(\mathrm{z}_{1}\right)\|T x-T y\| \leq a\|x-y\|, \\
& \left(\mathrm{z}_{2}\right)\|T x-T y\| \leq b(\|x-T x\|+\|y-T y\|), \\
& \left(\mathrm{z}_{3}\right)\|T x-T y\| \leq c(\|x-T y\|+\|y-T x\|) .
\end{aligned}
$$

Then $T$ has a unique fixed point $p$ and the Picard iteration $\left\{x_{n}\right\}_{n=0}^{\infty}$ defined by (1) converges to $p$, for any $x_{0} \in X$.

It is well known, see [11], that the conditions $\left(\mathrm{z}_{1}\right),\left(\mathrm{z}_{2}\right)$, and $\left(\mathrm{z}_{3}\right)$ are independent.

Let $x, y \in X$. Since $T$ satisfies condition $\mathrm{Z}$, at least one of the conditions from $\left(z_{1}\right),\left(z_{2}\right)$, and $\left(z_{3}\right)$ is satisfied. Then $T$ satisfies the inequalities

$$
\begin{aligned}
& \|T x-T y\| \leq \delta\|x-y\|+2 \delta\|x-T x\|, \\
& \|T x-T y\| \leq \delta\|x-y\|+2 \delta\|x-T y\|,
\end{aligned}
$$

for all $x, y \in X$ where $\delta:=\max \{a, b /(1-b), c /(1-c)\}, \delta \in$ $[0,1)$, and it was shown that this class of operators is wider than the class of Zamfirescu operators; see [12]. Any mapping satisfying condition (9) or (10) is called a quasi-contractive operator.

Osilike and Udomene [13] extended the previous definition by considering an operator satisfying the condition that there exist $L \geq 0$ and $\delta \in[0,1)$ such that, for all $x, y \in X$,

$$
\|T x-T y\| \leq \delta\|x-y\|+L\|x-T x\| .
$$

Thereafter, Imoru and Olatinwo [14] further generalized and extended the previous definition as follows: an operator $T$ is called contractive-like operator if there exist a constant $\delta \epsilon$ $[0,1)$ and a strictly increasing and continuous function $\varphi$ : $[0, \infty) \rightarrow[0, \infty)$ with $\varphi(0)=0$, such that, for each $x, y \in X$,

$$
\|T x-T y\| \leq \delta\|x-y\|+\varphi(\|x-T x\|) .
$$

Remark 1 (see [15]). A map satisfying (12) need not have a fixed point. However, using (12) it is obvious that if $T$ has a fixed point, then it is unique.

It is important to know whether an iterative scheme converges to fixed points of its associated map. In this context, there are numerous works dealing with the convergence of various iterative schemes in the literature, such as $[6,10,12$, 16-27].

As shown by Soltuz and Grosan [26, Theorem 3.1], in a real Banach space $X$, the Ishikawa iteration $\left\{x_{n}\right\}_{n=0}^{\infty}$ given by (3) converges to the fixed point of $T$, where $T: E \rightarrow E$ is a mapping satisfying condition (12).

It is known from [28, Corollary 2] that there is equivalence between convergence of iterative procedures (3), (5) and that of some other well-known iterative procedures for the class of operators satisfying (12).

Hussain et al. [29] introduced a Kirk-CR iterative scheme and proved the convergence of this iteration for the class of operators satisfying (12).

Remark 2. Putting $s=t=i=1$ in [29, Theorem 2.5], convergence of the CR iteration to a fixed point of contractive-like operators satisfying (12) can be obtained easily.

\section{Preliminaries}

Definition 3 (see [30]). Let $X$ be a Banach space and $T, \widetilde{T}$ : $X \rightarrow X$ two operators. We say that $\widetilde{T}$ is an approximate operator of $T$ if for all $x \in X$ and for a fixed $\varepsilon>0$ we have

$$
\|T x-\widetilde{T} x\| \leq \varepsilon
$$

Suppose that there exists a certain fixed point iteration that converges to some fixed point $p \in F_{T}$ and $\widetilde{T}$ has a fixed point $q \in F_{\widetilde{T}}$ which can be computed by certain method. If it cannot compute fixed point $p$ of $T$ due to various results, then approximate operator $\widetilde{T}$ can be used. One can find some of works done under this title in the following list $[15,24-$ $26,31]$.

In this paper, we prove the data dependence results for the multistep and CR iterative procedures utilizing the contractive-like operators satisfying (12).

The following lemma will be useful to prove the main results of this work.

Lemma 4 (see [26]). Let $\left\{a_{n}\right\}_{n=0}^{\infty}$ be a nonnegative sequence for which one assumes there exists $n_{0}(\epsilon) \in \mathbb{N}$, such that for all $n \geq n_{0}$ one has satisfied the inequality

$$
a_{n+1} \leq\left(1-\mu_{n}\right) a_{n}+\mu_{n} \eta_{n}
$$


where $\mu_{n} \in(0,1)$, for all $n \in \mathbb{N}, \sum_{n=0}^{\infty} \mu_{n}=\infty$ and $\eta_{n} \geq 0$, for all $n \in \mathbb{N}$. Then the following holds:

$$
0 \leq \lim _{n \rightarrow \infty} \sup a_{n} \leq \lim _{n \rightarrow \infty} \sup \eta_{n}
$$

\section{Main Results}

For simplicity we use the following notation through this section.

For any iterative process, $\left\{x_{n}\right\}_{n=0}^{\infty}$ and $\left\{u_{n}\right\}_{n=0}^{\infty}$ denote iterative sequences associated to $T$ and $\widetilde{T}$, respectively.

Theorem 5. Let $T: E \rightarrow E$ be a map satisfying (12) with $F_{T} \neq \emptyset$, and let $\widetilde{T}$ be an approximate operator of $T$ as in Definition 3. Let $\left\{x_{n}\right\}_{n=0}^{\infty},\left\{u_{n}\right\}_{n=0}^{\infty}$ be two iterative sequences defined by the multistep iteration (5) and with real sequences $\left\{\beta_{n}^{i}\right\}_{n=0}^{\infty} \subset[0,1), i=\overline{1, k-1}$, and $\left\{\alpha_{n}\right\}_{n=0}^{\infty} \subset[0,1)$ satisfying $\sum \alpha_{n}=\infty$. If $p=T p$ and $q=\widetilde{T} q$, then one has

$$
\|p-q\| \leq \frac{k \varepsilon}{1-\delta}
$$

Proof. For a given $x_{0} \in E$ and $u_{0} \in E$ we consider the following multistep iteration for $T$ and $\widetilde{T}$ :

$$
\begin{gathered}
x_{0} \in E, \\
x_{n+1}=\left(1-\alpha_{n}\right) x_{n}+\alpha_{n} T y_{n}^{1}, \quad n \in \mathbb{N}, \\
y_{n}^{i}=\left(1-\beta_{n}^{i}\right) x_{n}+\beta_{n}^{i} T y_{n}^{i+1}, \quad i=\overline{1, k-2,} \\
y_{n}^{k-1}=\left(1-\beta_{n}^{k-1}\right) x_{n}+\beta_{n}^{k-1} T x_{n}, \quad k \geq 2, \\
u_{0} \in E, \\
u_{n+1}=\left(1-\alpha_{n}\right) u_{n}+\alpha_{n} \widetilde{T} v_{n}^{1}, \quad n \in \mathbb{N}, \\
v_{n}^{i}=\left(1-\beta_{n}^{i}\right) u_{n}+\beta_{n}^{i} \widetilde{T} v_{n}^{i+1}, \quad i=\overline{1, k-2}, \\
v_{n}^{k-1}=\left(1-\beta_{n}^{k-1}\right) u_{n}+\beta_{n}^{k-1} \widetilde{T} u_{n}, \quad k \geq 2 .
\end{gathered}
$$

Then from (17), we get

$$
x_{n+1}-u_{n+1}=\left(1-\alpha_{n}\right)\left(x_{n}-u_{n}\right)+\alpha_{n}\left(T y_{n}^{1}-\widetilde{T} v_{n}^{1}\right) .
$$

Thus, we have the following estimates by using (18) and (12):

$$
\begin{aligned}
\| x_{n+1} & -u_{n+1} \| \\
& =\left\|\left(1-\alpha_{n}\right)\left(x_{n}-u_{n}\right)+\alpha_{n}\left(T y_{n}^{1}-\widetilde{T} v_{n}^{1}\right)\right\| \\
& \leq\left(1-\alpha_{n}\right)\left\|x_{n}-u_{n}\right\|+\alpha_{n}\left\|T y_{n}^{1}-\widetilde{T} v_{n}^{1}\right\| \\
& =\left(1-\alpha_{n}\right)\left\|x_{n}-u_{n}\right\|+\alpha_{n}\left\|T y_{n}^{1}-T v_{n}^{1}+T v_{n}^{1}-\widetilde{T} v_{n}^{1}\right\| \\
& \leq\left(1-\alpha_{n}\right)\left\|x_{n}-u_{n}\right\|+\alpha_{n}\left\|T y_{n}^{1}-T v_{n}^{1}\right\|
\end{aligned}
$$

$$
\begin{aligned}
& +\alpha_{n}\left\|T v_{n}^{1}-\widetilde{T} v_{n}^{1}\right\| \\
\leq & \left(1-\alpha_{n}\right)\left\|x_{n}-u_{n}\right\|+\alpha_{n} \delta\left\|y_{n}^{1}-v_{n}^{1}\right\| \\
& +\alpha_{n} \varphi\left(\left\|y_{n}^{1}-T y_{n}^{1}\right\|\right)+\alpha_{n} \varepsilon,
\end{aligned}
$$

$$
\begin{aligned}
\| y_{n}^{1}- & v_{n}^{1} \| \\
= & \left\|\left(1-\beta_{n}^{1}\right)\left(x_{n}-u_{n}\right)+\beta_{n}^{1}\left(T y_{n}^{2}-\widetilde{T} v_{n}^{2}\right)\right\| \\
\leq & \left(1-\beta_{n}^{1}\right)\left\|x_{n}-u_{n}\right\|+\beta_{n}^{1}\left\|T y_{n}^{2}-\widetilde{T} v_{n}^{2}\right\| \\
\leq & \left(1-\beta_{n}^{1}\right)\left\|x_{n}-u_{n}\right\|+\beta_{n}^{1}\left\|T y_{n}^{2}-T v_{n}^{2}\right\| \\
& +\beta_{n}^{1}\left\|T v_{n}^{2}-\widetilde{T} v_{n}^{2}\right\| \\
\leq & \left(1-\beta_{n}^{1}\right)\left\|x_{n}-u_{n}\right\|+\beta_{n}^{1} \delta\left\|y_{n}^{2}-v_{n}^{2}\right\| \\
& +\beta_{n}^{1} \varphi\left(\left\|y_{n}^{2}-T y_{n}^{2}\right\|\right)+\beta_{n}^{1} \varepsilon, \\
\| y_{n}^{2}- & v_{n}^{2} \| \\
= & \left\|\left(1-\beta_{n}^{2}\right)\left(x_{n}-u_{n}\right)+\beta_{n}^{2}\left(T y_{n}^{3}-\widetilde{T} v_{n}^{3}\right)\right\| \\
\leq & \left(1-\beta_{n}^{2}\right)\left\|x_{n}-u_{n}\right\|+\beta_{n}^{2}\left\|T y_{n}^{3}-\widetilde{T} v_{n}^{3}\right\| \\
\leq & \left(1-\beta_{n}^{2}\right)\left\|x_{n}-u_{n}\right\|+\beta_{n}^{2}\left\|T y_{n}^{3}-T v_{n}^{3}\right\| \\
& +\beta_{n}^{2}\left\|T v_{n}^{3}-\widetilde{T} v_{n}^{3}\right\| \\
\leq & \left(1-\beta_{n}^{2}\right)\left\|x_{n}-u_{n}\right\|+\beta_{n}^{2} \delta\left\|y_{n}^{3}-v_{n}^{3}\right\| \\
& +\beta_{n}^{2} \varphi\left(\left\|y_{n}^{3}-T y_{n}^{3}\right\|\right)+\beta_{n}^{2} \varepsilon,
\end{aligned}
$$

$\left\|y_{n}^{3}-v_{n}^{3}\right\|$

$$
\begin{aligned}
= & \left\|\left(1-\beta_{n}^{3}\right)\left(x_{n}-u_{n}\right)+\beta_{n}^{3}\left(T y_{n}^{4}-\widetilde{T} v_{n}^{4}\right)\right\| \\
\leq & \left(1-\beta_{n}^{3}\right)\left\|x_{n}-u_{n}\right\|+\beta_{n}^{3}\left\|T y_{n}^{4}-\widetilde{T} v_{n}^{4}\right\| \\
\leq & \left(1-\beta_{n}^{3}\right)\left\|x_{n}-u_{n}\right\|+\beta_{n}^{3}\left\|T y_{n}^{4}-T v_{n}^{4}\right\| \\
& +\beta_{n}^{3}\left\|T v_{n}^{4}-\widetilde{T} v_{n}^{4}\right\| \\
\leq & \left(1-\beta_{n}^{3}\right)\left\|x_{n}-u_{n}\right\|+\beta_{n}^{3} \delta\left\|y_{n}^{4}-v_{n}^{4}\right\| \\
& +\beta_{n}^{3} \varphi\left(\left\|y_{n}^{4}-T y_{n}^{4}\right\|\right)+\beta_{n}^{3} \varepsilon .
\end{aligned}
$$

Combining (19), (20), (21), and (22) we obtain

$$
\begin{aligned}
\| x_{n+1}- & u_{n+1} \| \\
\leq & {\left[\left(1-\alpha_{n}\right)+\alpha_{n} \delta\left(1-\beta_{n}^{1}\right)+\alpha_{n} \delta^{2} \beta_{n}^{1}\left(1-\beta_{n}^{2}\right)\right.} \\
& \left.+\alpha_{n} \delta^{3} \beta_{n}^{1} \beta_{n}^{2}\left(1-\beta_{n}^{3}\right)\right]\left\|x_{n}-u_{n}\right\| \\
& +\alpha_{n} \delta^{4} \beta_{n}^{1} \beta_{n}^{2} \beta_{n}^{3}\left\|y_{n}^{4}-v_{n}^{4}\right\|
\end{aligned}
$$




$$
\begin{aligned}
& +\alpha_{n} \delta^{3} \beta_{n}^{1} \beta_{n}^{2} \beta_{n}^{3} \varphi\left(\left\|y_{n}^{4}-T y_{n}^{4}\right\|\right)+\alpha_{n} \delta^{3} \beta_{n}^{1} \beta_{n}^{2} \beta_{n}^{3} \varepsilon \\
& +\alpha_{n} \delta^{2} \beta_{n}^{1} \beta_{n}^{2} \varphi\left(\left\|y_{n}^{3}-T y_{n}^{3}\right\|\right)+\alpha_{n} \delta^{2} \beta_{n}^{1} \beta_{n}^{2} \varepsilon \\
& +\alpha_{n} \delta \beta_{n}^{1} \varphi\left(\left\|y_{n}^{2}-T y_{n}^{2}\right\|\right)+\alpha_{n} \delta \beta_{n}^{1} \varepsilon \\
& +\alpha_{n} \varphi\left(\left\|y_{n}^{1}-T y_{n}^{1}\right\|\right)+\alpha_{n} \varepsilon .
\end{aligned}
$$

Thus, inductively, we get

$$
\begin{aligned}
\| x_{n+1}- & u_{n+1} \| \\
\leq & {\left[\left(1-\alpha_{n}\right)+\delta \alpha_{n}\left(1-\beta_{n}^{1}\right)+\delta^{2} \alpha_{n} \beta_{n}^{1}\left(1-\beta_{n}^{2}\right)\right.} \\
& +\delta^{3} \alpha_{n} \beta_{n}^{1} \beta_{n}^{2}\left(1-\beta_{n}^{3}\right)+\delta^{4} \alpha_{n} \beta_{n}^{1} \beta_{n}^{2} \beta_{n}^{3}\left(1-\beta_{n}^{4}\right) \\
& \left.+\cdots+\delta^{k-2} \alpha_{n} \beta_{n}^{1} \beta_{n}^{2} \cdots \beta_{n}^{k-3}\left(1-\beta_{n}^{k-2}\right)\right]\left\|x_{n}-u_{n}\right\| \\
+ & \delta^{k-1} \alpha_{n} \beta_{n}^{1} \beta_{n}^{2} \cdots \beta_{n}^{k-2}\left\|y_{n}^{k-1}-v_{n}^{k-1}\right\| \\
+ & \delta^{k-2} \alpha_{n} \beta_{n}^{1} \beta_{n}^{2} \cdots \beta_{n}^{k-2} \varphi\left(\left\|y_{n}^{k-1}-T y_{n}^{k-1}\right\|\right) \\
+ & \cdots+\delta^{3} \alpha_{n} \beta_{n}^{1} \beta_{n}^{2} \beta_{n}^{3} \varphi\left(\left\|y_{n}^{4}-T y_{n}^{4}\right\|\right) \\
+ & \delta^{2} \alpha_{n} \beta_{n}^{1} \beta_{n}^{2} \varphi\left(\left\|y_{n}^{3}-T y_{n}^{3}\right\|\right) \\
+ & \delta \alpha_{n} \beta_{n}^{1} \varphi\left(\left\|y_{n}^{2}-T y_{n}^{2}\right\|\right)+\alpha_{n} \varphi\left(\left\|y_{n}^{1}-T y_{n}^{1}\right\|\right) \\
+ & \delta^{k-2} \alpha_{n} \beta_{n}^{1} \beta_{n}^{2} \cdots \beta_{n}^{k-2} \varepsilon+\cdots+\delta^{2} \alpha_{n} \beta_{n}^{1} \beta_{n}^{2} \varepsilon \\
+ & \delta \alpha_{n} \beta_{n}^{1} \varepsilon+\alpha_{n} \varepsilon .
\end{aligned}
$$

Using now (17) and (12), we get

$$
\begin{aligned}
\| y_{n}^{k-1}- & v_{n}^{k-1} \| \\
= & \left\|\left(1-\beta_{n}^{k-1}\right)\left(x_{n}-u_{n}\right)+\beta_{n}^{k-1}\left(T x_{n}-\widetilde{T} u_{n}\right)\right\| \\
\leq & \left(1-\beta_{n}^{k-1}\right)\left\|x_{n}-u_{n}\right\|+\beta_{n}^{k-1}\left\|T x_{n}-\widetilde{T} u_{n}\right\| \\
\leq & \left(1-\beta_{n}^{k-1}\right)\left\|x_{n}-u_{n}\right\|+\beta_{n}^{k-1}\left\|T x_{n}-T u_{n}\right\| \\
& +\beta_{n}^{k-1}\left\|T u_{n}-\widetilde{T} u_{n}\right\| \\
\leq & \left(1-\beta_{n}^{k-1}\right)\left\|x_{n}-u_{n}\right\|+\beta_{n}^{k-1} \delta\left\|x_{n}-u_{n}\right\| \\
& +\beta_{n}^{k-1} \varphi\left(\left\|x_{n}-T x_{n}\right\|\right)+\beta_{n}^{k-1} \varepsilon .
\end{aligned}
$$

Substituting (25) in (24) we have

$$
\begin{aligned}
& \left\|x_{n+1}-u_{n+1}\right\| \\
& \leq\left[\left(1-\alpha_{n}\right)+\delta \alpha_{n}\left(1-\beta_{n}^{1}\right)+\delta^{2} \alpha_{n} \beta_{n}^{1}\left(1-\beta_{n}^{2}\right)\right. \\
& \quad+\delta^{3} \alpha_{n} \beta_{n}^{1} \beta_{n}^{2}\left(1-\beta_{n}^{3}\right)+\delta^{4} \alpha_{n} \beta_{n}^{1} \beta_{n}^{2} \beta_{n}^{3}\left(1-\beta_{n}^{4}\right) \\
& +\cdots+\delta^{k-2} \alpha_{n} \beta_{n}^{1} \beta_{n}^{2} \cdots \beta_{n}^{k-3}\left(1-\beta_{n}^{k-2}\right)
\end{aligned}
$$

$$
\begin{aligned}
& +\delta^{k-1} \alpha_{n} \beta_{n}^{1} \beta_{n}^{2} \cdots \beta_{n}^{k-2}\left(1-\beta_{n}^{k-1}\right) \\
& \left.+\delta^{k} \alpha_{n} \beta_{n}^{1} \beta_{n}^{2} \cdots \beta_{n}^{k-2} \beta_{n}^{k-1}\right]\left\|x_{n}-u_{n}\right\| \\
+ & \delta^{k-1} \alpha_{n} \beta_{n}^{1} \beta_{n}^{2} \cdots \beta_{n}^{k-2} \beta_{n}^{k-1} \varphi\left(\left\|x_{n}-T x_{n}\right\|\right) \\
+ & \delta^{k-2} \alpha_{n} \beta_{n}^{1} \beta_{n}^{2} \cdots \beta_{n}^{k-2} \varphi\left(\left\|y_{n}^{k-1}-T y_{n}^{k-1}\right\|\right) \\
+ & +\delta^{3} \alpha_{n} \beta_{n}^{1} \beta_{n}^{2} \beta_{n}^{3} \varphi\left(\left\|y_{n}^{4}-T y_{n}^{4}\right\|\right) \\
+ & \delta^{2} \alpha_{n} \beta_{n}^{1} \beta_{n}^{2} \varphi\left(\left\|y_{n}^{3}-T y_{n}^{3}\right\|\right) \\
+ & \delta \alpha_{n} \beta_{n}^{1} \varphi\left(\left\|y_{n}^{2}-T y_{n}^{2}\right\|\right)+\alpha_{n} \varphi\left(\left\|y_{n}^{1}-T y_{n}^{1}\right\|\right) \\
+ & \delta^{k-1} \alpha_{n} \beta_{n}^{1} \beta_{n}^{2} \cdots \beta_{n}^{k-2} \beta_{n}^{k-1} \varepsilon \\
+ & \delta^{k-2} \alpha_{n} \beta_{n}^{1} \beta_{n}^{2} \cdots \beta_{n}^{k-2} \varepsilon \\
+ & \cdots+\delta^{2} \alpha_{n} \beta_{n}^{1} \beta_{n}^{2} \varepsilon+\delta \alpha_{n} \beta_{n}^{1} \varepsilon+\alpha_{n} \varepsilon .
\end{aligned}
$$

If this inequality is rearranged using $\left\{\beta_{n}^{i}\right\}_{n=0}^{\infty} \subset[0,1), \delta^{i+1}<$ $\delta^{i}$ for each $i=\overline{1, k-1}$, then we get the following inequality as follows:

$$
\begin{aligned}
\| x_{n+1}- & u_{n+1} \| \\
\leq & {\left[1-\alpha_{n}(1-\delta)\right]\left\|x_{n}-u_{n}\right\| } \\
& +\alpha_{n}(1-\delta) \frac{\varphi\left(\left\|x_{n}-T x_{n}\right\|\right)}{1-\delta} \\
& +\alpha_{n}(1-\delta) \\
& \times \frac{\left\{\varphi\left(\left\|y_{n}^{k-1}-T y_{n}^{k-1}\right\|\right)+\cdots+\varphi\left(\left\|y_{n}^{1}-T y_{n}^{1}\right\|\right)+k \varepsilon\right\}}{1-\delta} .
\end{aligned}
$$

Denote

$$
\begin{aligned}
a_{n}:=\left\|x_{n}-u_{n}\right\|, \\
\mu_{n}:=\alpha_{n}(1-\delta) \in(0.1), \\
\eta_{n}:=\frac{\varphi\left(\left\|x_{n}-T x_{n}\right\|\right)}{1-\delta} \\
\quad+\frac{\varphi\left(\left\|y_{n}^{k-1}-T y_{n}^{k-1}\right\|\right)+\cdots+\varphi\left(\left\|y_{n}^{1}-T y_{n}^{1}\right\|\right)+k \varepsilon}{1-\delta} .
\end{aligned}
$$

From [26, Theorem 3.1] and [28, Corollary 2] we have $\lim _{n \rightarrow \infty}\left\|x_{n}-p\right\|=0$. Since $T$ satisfies condition (12), and $p \in F_{T}$, that is, $T p=p$, it follows from (12) that

$$
\begin{aligned}
0 & \leq\left\|x_{n}-T x_{n}\right\| \\
& \leq\left\|x_{n}-p\right\|+\left\|T p-T x_{n}\right\| \\
& \leq\left\|x_{n}-p\right\|+\delta\left\|p-x_{n}\right\|+\varphi(\|p-T p\|) \\
& =(1+\delta)\left\|x_{n}-p\right\| \longrightarrow 0 \quad \text { as } n \longrightarrow \infty .
\end{aligned}
$$


Considering $\beta_{n}^{i} \in[0,1)$, for all $n \in \mathbb{N}, i=\overline{1, k-1}$, and using (12) and (5) we have

$$
\begin{aligned}
0 \leq & \left\|y_{n}^{1}-T y_{n}^{1}\right\|=\left\|y_{n}^{1}-p+p-T y_{n}^{1}\right\| \\
\leq & \left\|y_{n}^{1}-p\right\|+\left\|T p-T y_{n}^{1}\right\| \\
\leq & \left\|y_{n}^{1}-p\right\|+\delta\left\|p-y_{n}^{1}\right\|+\varphi(\|p-T p\|) \\
= & (1+\delta)\left\|y_{n}^{1}-p\right\| \\
= & (1+\delta)\left\|\left(1-\beta_{n}^{1}\right) y_{n}^{2}+\beta_{n}^{1} T y_{n}^{2}-p\left(1-\beta_{n}^{1}+\beta_{n}^{1}\right)\right\| \\
\leq & (1+\delta)\left\{\left(1-\beta_{n}^{1}\right)\left\|y_{n}^{2}-p\right\|+\beta_{n}^{1}\left\|T y_{n}^{2}-T p\right\|\right\} \\
\leq & (1+\delta)\left\{\left(1-\beta_{n}^{1}\right)\left\|y_{n}^{2}-p\right\|+\beta_{n}^{1} \delta\left\|y_{n}^{2}-p\right\|\right\} \\
= & (1+\delta)\left[1-\beta_{n}^{1}(1-\delta)\right]\left\|y_{n}^{2}-p\right\| \\
= & (1+\delta)\left[1-\beta_{n}^{1}(1-\delta)\right] \\
& \times\left\|\left(1-\beta_{n}^{2}\right) y_{n}^{3}+\beta_{n}^{2} T y_{n}^{3}-p\left(1-\beta_{n}^{2}+\beta_{n}^{2}\right)\right\| \\
\leq & (1+\delta)\left[1-\beta_{n}^{1}(1-\delta)\right] \\
& \times\left\{\left(1-\beta_{n}^{2}\right)\left\|y_{n}^{3}-p\right\|+\beta_{n}^{2}\left\|T y_{n}^{3}-T p\right\|\right\} \\
\leq & (1+\delta)\left[1-\beta_{n}^{1}(1-\delta)\right] \\
& \times\left[1-\beta_{n}^{2}(1-\delta)\right]\left\|y_{n}^{3}-p\right\| \\
& \vdots \\
& \cdots\left[1-\beta_{n}^{k-1}(1-\delta)\right]\left\|x_{n}-p\right\| \\
\leq & (1+\delta)\left\|x_{n}-p\right\| \longrightarrow 0 \quad \text { as } n \longrightarrow \infty . \\
& \cdots\left[1-\beta_{n}^{k-2}(1-\delta)\right]\left\|y_{n}^{k-1}-p\right\| \\
& (1+\delta)\left[1-\beta_{n}^{1}(1-\delta)\right] \\
& \\
& (1+\delta)] \\
&
\end{aligned}
$$

It is easy to see from (30) that this result is also valid for $\| T y_{n}^{2}-$ $y_{n}^{2}\|, \ldots\|, T y_{n}^{k-1}-y_{n}^{k-1} \|$.

Making use of the fact that $\varphi$ is a continuous map we have

$$
\begin{aligned}
\lim _{n \rightarrow \infty} \varphi\left(\left\|x_{n}-T x_{n}\right\|\right) & =\lim _{n \rightarrow \infty} \varphi\left(\left\|y_{n}^{1}-T y_{n}^{1}\right\|\right) \\
& =\cdots=\lim _{n \rightarrow \infty} \varphi\left(\left\|y_{n}^{k-1}-T y_{n}^{k-1}\right\|\right)=0 .
\end{aligned}
$$

Hence an application of Lemma 4 to (27) leads to

$$
\|p-q\| \leq \frac{k \varepsilon}{1-\delta} .
$$

Now we prove result on data dependence for the CR iterative procedure.

Theorem 6. Let $T: E \rightarrow E$ be a map satisfying (12) with $F_{T} \neq \emptyset$, and let $\widetilde{T}$ be an approximate operator of $T$ as in Definition 3. Let $\left\{x_{n}\right\}_{n=0}^{\infty},\left\{u_{n}\right\}_{n=0}^{\infty}$ be two iterative sequences defined by the CR iteration (6) and with real sequences $\left\{\beta_{n}\right\}_{n=0}^{\infty}$, $\left\{\mu_{n}\right\}_{n=0}^{\infty},\left\{\alpha_{n}\right\}_{n=0}^{\infty} \subset[0,1]$ satisfying (i) $1 / 2 \leq \alpha_{n}$, for all $n \in \mathbb{N}$, and (ii) $\sum_{n=0}^{\infty} \alpha_{n}=\infty$. If $p=T p$ and $q=\widetilde{T} q$, then one has

$$
\|p-q\| \leq \frac{5 \varepsilon}{1-\delta} .
$$

Proof. For a given $x_{0} \in E$ and $u_{0} \in E$ we consider the following iteration for $T$ and $\widetilde{T}$ :

$$
\begin{aligned}
& x_{n+1}=\left(1-\alpha_{n}\right) y_{n}+\alpha_{n} T y_{n}, \\
& y_{n}=\left(1-\beta_{n}\right) T x_{n}+\beta_{n} T z_{n}, \\
& z_{n}=\left(1-\mu_{n}\right) x_{n}+\mu_{n} T x_{n}, \\
& u_{n+1}=\left(1-\alpha_{n}\right) v_{n}+\alpha_{n} \widetilde{T} v_{n}, \\
& v_{n}=\left(1-\beta_{n}\right) \widetilde{T} u_{n}+\beta_{n} \widetilde{T} w_{n}, \\
& w_{n}=\left(1-\mu_{n}\right) u_{n}+\mu_{n} \widetilde{T} u_{n} .
\end{aligned}
$$

Then from (34) we have

$$
\begin{gathered}
x_{n+1}-u_{n+1}=\left(1-\alpha_{n}\right)\left(y_{n}-v_{n}\right)+\alpha_{n}\left(T y_{n}-\widetilde{T} v_{n}\right), \\
y_{n}-v_{n}=\left(1-\beta_{n}\right)\left(T x_{n}-\widetilde{T} u_{n}\right)+\beta_{n}\left(T z_{n}-\widetilde{T} w_{n}\right), \\
z_{n}-w_{n}=\left(1-\mu_{n}\right)\left(x_{n}-u_{n}\right)+\mu_{n}\left(T x_{n}-\widetilde{T} u_{n}\right) .
\end{gathered}
$$

Thus, by considering (35), it follows from (6) and (12) that

$$
\begin{aligned}
\| x_{n+1} & -u_{n+1} \| \\
= & \left\|\left(1-\alpha_{n}\right)\left(y_{n}-v_{n}\right)+\alpha_{n}\left(T y_{n}-\widetilde{T} v_{n}\right)\right\| \\
\leq & \left(1-\alpha_{n}\right)\left\|y_{n}-v_{n}\right\|+\alpha_{n}\left\|T y_{n}-\widetilde{T} v_{n}\right\| \\
= & \left(1-\alpha_{n}\right)\left\|y_{n}-v_{n}\right\|+\alpha_{n}\left\|T y_{n}-T v_{n}+T v_{n}-\widetilde{T} v_{n}\right\| \\
\leq & \left(1-\alpha_{n}\right)\left\|y_{n}-v_{n}\right\|+\alpha_{n}\left\|T y_{n}-T v_{n}\right\| \\
& +\alpha_{n}\left\|T v_{n}-\widetilde{T} v_{n}\right\| \\
\leq & \left(1-\alpha_{n}\right)\left\|y_{n}-v_{n}\right\|+\alpha_{n} \delta\left\|y_{n}-v_{n}\right\| \\
& +\alpha_{n} \varphi\left(\left\|y_{n}-T y_{n}\right\|\right)+\alpha_{n} \varepsilon
\end{aligned}
$$




$$
\begin{aligned}
= & {\left[1-\alpha_{n}(1-\delta)\right]\left\|y_{n}-v_{n}\right\| } \\
& +\alpha_{n} \varphi\left(\left\|y_{n}-T y_{n}\right\|\right)+\alpha_{n} \varepsilon,
\end{aligned}
$$

$$
\begin{aligned}
\| y_{n}- & v_{n} \| \\
= & \left\|\left(1-\beta_{n}\right)\left(T x_{n}-\widetilde{T} u_{n}\right)+\beta_{n}\left(T z_{n}-\widetilde{T} w_{n}\right)\right\| \\
\leq & \left(1-\beta_{n}\right)\left\|T x_{n}-\widetilde{T} u_{n}\right\|+\beta_{n}\left\|T z_{n}-\widetilde{T} w_{n}\right\| \\
= & \left(1-\beta_{n}\right)\left\|T x_{n}-T u_{n}+T u_{n}-\widetilde{T} u_{n}\right\| \\
& +\beta_{n}\left\|T z_{n}-T w_{n}+T w_{n}-\widetilde{T} w_{n}\right\| \\
\leq & \left(1-\beta_{n}\right)\left\{\left\|T x_{n}-T u_{n}\right\|+\left\|T u_{n}-\widetilde{T} u_{n}\right\|\right\} \\
& +\beta_{n}\left\{\left\|T z_{n}-T w_{n}\right\|+\left\|T w_{n}-\widetilde{T} w_{n}\right\|\right\} \\
\leq & \left(1-\beta_{n}\right) \delta\left\|x_{n}-u_{n}\right\|+\beta_{n} \delta\left\|z_{n}-w_{n}\right\| \\
& +\left(1-\beta_{n}\right) \varphi\left(\left\|x_{n}-T x_{n}\right\|\right) \\
& +\left(1-\beta_{n}\right) \varepsilon+\beta_{n} \varphi\left(\left\|T z_{n}-T w_{n}\right\|\right)+\beta_{n} \varepsilon
\end{aligned}
$$

$$
\begin{aligned}
\| z_{n}- & w_{n} \| \\
= & \left\|\left(1-\mu_{n}\right)\left(x_{n}-u_{n}\right)+\mu_{n}\left(T x_{n}-\widetilde{T} u_{n}\right)\right\| \\
\leq & \left(1-\mu_{n}\right)\left\|x_{n}-u_{n}\right\|+\mu_{n}\left\|T x_{n}-\widetilde{T} u_{n}\right\| \\
= & \left(1-\mu_{n}\right)\left\|x_{n}-u_{n}\right\|+\mu_{n}\left\|T x_{n}-T u_{n}+T u_{n}-\widetilde{T} u_{n}\right\| \\
\leq & \left(1-\mu_{n}\right)\left\|x_{n}-u_{n}\right\| \\
& +\mu_{n}\left\{\left\|T x_{n}-T u_{n}\right\|+\left\|T u_{n}-\widetilde{T} u_{n}\right\|\right\} \\
\leq & \left(1-\mu_{n}\right)\left\|x_{n}-u_{n}\right\| \\
& +\mu_{n}\left\{\delta\left\|x_{n}-u_{n}\right\|+\varphi\left(\left\|x_{n}-T x_{n}\right\|\right)+\varepsilon\right\} \\
= & {\left[1-\mu_{n}(1-\delta)\right]\left\|x_{n}-u_{n}\right\| } \\
& +\mu_{n} \varphi\left(\left\|x_{n}-T x_{n}\right\|\right)+\mu_{n} \varepsilon .
\end{aligned}
$$

Combining (36), (37), and (38) we obtain

$$
\begin{aligned}
\| x_{n+1}- & u_{n+1} \| \\
\leq & {\left[1-\alpha_{n}(1-\delta)\right] } \\
& \times\left[\left(1-\beta_{n}\right) \delta+\beta_{n} \delta\left[1-\mu_{n}(1-\delta)\right]\right]\left\|x_{n}-u_{n}\right\| \\
+ & {\left[1-\alpha_{n}(1-\delta)\right] } \\
& \times\left\{\left[1-\beta_{n}\left(1-\delta \mu_{n}\right)\right] \varphi\left(\left\|x_{n}-T x_{n}\right\|\right)+\beta_{n} \mu_{n} \delta \varepsilon\right. \\
& \left.\quad+\left(1-\beta_{n}\right) \varepsilon+\beta_{n} \varphi\left(\left\|z_{n}-T z_{n}\right\|\right)+\beta_{n} \varepsilon\right\} \\
+ & \alpha_{n} \varphi\left(\left\|y_{n}-T y_{n}\right\|\right)+\alpha_{n} \varepsilon .
\end{aligned}
$$

It may be noted that for $\left\{\beta_{n}\right\}_{n=0}^{\infty},\left\{\mu_{n}\right\}_{n=0}^{\infty} \subset[0,1)$, and $\delta \in[0,1)$ the following inequalities are always true:

$$
1-\mu_{n}(1-\delta)<1, \quad \beta_{n} \mu_{n} \delta<1, \quad \delta \mu_{n}<\delta .
$$

Using now the inequality $\delta \mu_{n}<\delta$ we get

$$
1-\beta_{n}\left(1-\delta \mu_{n}\right)<1-\beta_{n}(1-\delta)<1 .
$$

Therefore an application of (40) and (41) to (39) gives us

$$
\begin{aligned}
\| x_{n+1}- & u_{n+1} \| \\
\leq & {\left[1-\alpha_{n}(1-\delta)\right]\left\|x_{n}-u_{n}\right\| } \\
& +\left[1-\alpha_{n}(1-\delta)\right] \\
& \times\left\{\varphi\left(\left\|x_{n}-T x_{n}\right\|\right)+\varepsilon+\varepsilon+\varphi\left(\left\|z_{n}-T z_{n}\right\|\right)\right\} \\
& +\alpha_{n} \varphi\left(\left\|y_{n}-T y_{n}\right\|\right)+\alpha_{n} \varepsilon .
\end{aligned}
$$

Now, by the condition (i) $1 / 2 \leq \alpha_{n}$, for all $n \in \mathbb{N}$ we have

$$
1-\alpha_{n} \leq \alpha_{n} \text {. }
$$

Utilizing (43) in (42) we obtain

$$
\begin{aligned}
\| x_{n+1}- & u_{n+1} \| \\
\leq & {\left[1-\alpha_{n}(1-\delta)\right]\left\|x_{n}-u_{n}\right\| } \\
& +\alpha_{n}(1-\delta) \frac{\left\{2 \varphi\left(\left\|x_{n}-T x_{n}\right\|\right)+\varphi\left(\left\|y_{n}-T y_{n}\right\|\right)\right\}}{1-\delta} \\
& +\alpha_{n}(1-\delta) \frac{\left\{2 \varphi\left(\left\|z_{n}-T z_{n}\right\|\right)+5 \varepsilon\right\}}{1-\delta} .
\end{aligned}
$$

Denote

$$
\begin{gathered}
a_{n}:=\left\|x_{n}-u_{n}\right\|, \\
\mu_{n}:=\alpha_{n}(1-\delta) \in(0,1), \\
\eta_{n}:=\frac{\left\{2 \varphi\left(\left\|x_{n}-T x_{n}\right\|\right)+\varphi\left(\left\|y_{n}-T y_{n}\right\|\right)+2 \varphi\left(\left\|z_{n}-T z_{n}\right\|\right)+5 \varepsilon\right\}}{1-\delta} .
\end{gathered}
$$

From Remark 2, we have $\lim _{n \rightarrow \infty}\left\|x_{n}-p\right\|=0$. Since $T$ satisfies condition (12), and $p \in F_{T}$, that is, $T p=p$, using similar arguments as in the proof of Theorem 5 , we get

$$
\lim _{n \rightarrow \infty}\left\|x_{n}-T x_{n}\right\|=\lim _{n \rightarrow \infty}\left\|y_{n}-T y_{n}\right\|=\lim _{n \rightarrow \infty}\left\|z_{n}-T z_{n}\right\|=0 .
$$

Making use of the fact that $\varphi$ is a continuous map we have

$$
\begin{aligned}
\lim _{n \rightarrow \infty} \varphi\left(\left\|x_{n}-T x_{n}\right\|\right) & =\lim _{n \rightarrow \infty} \varphi\left(\left\|y_{n}-T y_{n}\right\|\right) \\
& =\lim _{n \rightarrow \infty} \varphi\left(\left\|z_{n}-T z_{n}\right\|\right)=0 .
\end{aligned}
$$

Hence an application of Lemma 4 to (44) leads to

$$
\|p-q\| \leq \frac{5 \varepsilon}{1-\delta} .
$$


Corollary 7. Since the Mann (2), Ishikawa (3), and Noor (4) iterative processes are special cases of the multistep iterative scheme (5), the data dependence results of these iterative processes can be obtained similarly.

\section{References}

[1] E. Picard, "Memoire sur la theorie des equations aux derivees partielles et la methode des approximations successives," Journal de Mathématiques Pures et Appliquées, vol. 6, pp. 145-210, 1890.

[2] W. R. Mann, "Mean value methods in iteration," Proceedings of the American Mathematical Society, vol. 4, pp. 506-510, 1953.

[3] M. A. Krasnoselkij, "Two remarks on the method of successive approximations," Uspekhi Matematicheskikh Nauk, vol. 10, no. 1(63), pp. 123-127, 1955.

[4] S. Ishikawa, "Fixed points by a new iteration method," Proceedings of the American Mathematical Society, vol. 44, pp. 147-150, 1974.

[5] M. A. Noor, "New approximation schemes for general variational inequalities," Journal of Mathematical Analysis and Applications, vol. 251, no. 1, pp. 217-229, 2000.

[6] B. E. Rhoades and S. M. Soltuz, "The equivalence between Mann-Ishikawa iterations and multistep iteration," Nonlinear Analysis, vol. 58, no. 1-2, pp. 219-228, 2004.

[7] R. Chugh, V. Kumar, and S. Kumar, "Strong converge of a new three step iterative scheme in Banach spaces," American Journal of Computational Mathematics, vol. 2, pp. 345-357, 2012.

[8] R. Kannan, "Some results on fixed points," Bulletin of the Calcutta Mathematical Society, vol. 60, pp. 71-76, 1968.

[9] S. K. Chatterjea, "Fixed-point theorems," Doklady Bolgarskor Akademii Nauk, vol. 25, pp. 727-730, 1972.

[10] T. Zamfirescu, "Fix point theorems in metric spaces," Archiv der Mathematik, vol. 23, pp. 292-298, 1972.

[11] B. E. Rhoades, "A comparison of various definitions of contractive mappings," Transactions of the American Mathematical Society, vol. 226, pp. 257-290, 1977.

[12] V. Berinde, "On the convergence of the Ishikawa iteration in the class of quasi contractive operators," Acta Mathematica Universitatis Comenianae, vol. 73, no. 1, pp. 119-126, 2004.

[13] M. O. Osilike and A. Udomene, "Short proofs of stability results for fixed point iteration procedures for a class of contractivetype mappings," Indian Journal of Pure and Applied Mathematics, vol. 30, no. 12, pp. 1229-1234, 1999.

[14] C. O. Imoru and M. O. Olatinwo, "On the stability of Picard and Mann iteration processes," Carpathian Journal of Mathematics, vol. 19, no. 2, pp. 155-160, 2003.

[15] F. Gursoy, V. Karakaya, and B. E. Rhoades, "Data dependence results of new multistep and S-iterative schemes for contractivelike operators," Fixed Point Theory and Applications, vol. 2013, article 76, 2013.

[16] A. Rafiq, "On the convergence of the three-step iteration process in the class of quasi-contractive operators," Acta Mathematica Academiae Paedagogicae Nyiregyhaziensis, vol. 22, no. 3, pp. 305-309, 2006.

[17] B. E. Rhoades, "Fixed point theorems and stability results for fixed point iteration procedures. II," Indian Journal of Pure and Applied Mathematics, vol. 24, no. 11, pp. 691-703, 1993.

[18] I. A. Rus, Generalized Contractions and Applications, Cluj University Press, Cluj-Napoca, Romania, 2001.
[19] İ. Yildirim, M. Özdemir, and H. Kiziltunç, “On the convergence of a new two-step iteration in the class of quasi-contractive operators," International Journal of Mathematical Analysis, vol. 3, no. 37-40, pp. 1881-1892, 2009.

[20] J. A. Park, "Mann-iteration process for the fixed point of strictly pseudocontractive mapping in some Banach spaces," Journal of the Korean Mathematical Society, vol. 31, no. 3, pp. 333-337, 1994.

[21] M. Ertürk and V. Karakaya, "n-tuplet fixed point theorems for contractive type mappings in partially ordered metric spaces," Journal of Inequalities and Applications, vol. 2013, article 196, 2013.

[22] W. Phuengrattana and S. Suantai, "On the rate of convergence of Mann, Ishikawa, Noor and SP-iterations for continuous functions on an arbitrary interval," Journal of Computational and Applied Mathematics, vol. 235, no. 9, pp. 3006-3014, 2011.

[23] R. Chugh and V. Kumar, "Strong convergence of SP iterative scheme for quasi-contractive operators in Banach spaces," International Journal of Computer Applications, vol. 31, no. 5, pp. 21-27, 2011.

[24] S. M. Soltuz, "Data dependence for Mann iteration," Octogon Mathematical Magazine, vol. 9, pp. 825-828, 2001.

[25] S. M. Soltuz, "Data dependence for Ishikawa iteration," Lecturas Matemáticas, vol. 25, no. 2, pp. 149-155, 2004.

[26] S. M. Soltuz and T. Grosan, "Data dependence for Ishikawa iteration when dealing with contractive-like operators," Fixed Point Theory and Applications, vol. 2008, Article ID 242916, 7 pages, 2008.

[27] S. M. Soltuz, "The equivalence between Krasnoselskij, Mann, Ishikawa, Noor and multistep iterations," Mathematical Communications, vol. 12, no. 1, pp. 53-61, 2007.

[28] F. Gürsoy, V. Karakaya, and B. E. Rhoades, “The equivalence among new multistep iteration, S-iteration and some other iterative schemes," http://arxiv.org/abs/1211.5701 .

[29] N. Hussain, R. Chugh, V. Kumar, and A. Rafiq, "On the rate of convergence of Kirk-type iterative schemes," Journal of Applied Mathematics, vol. 2012, Article ID 526503, 22 pages, 2012.

[30] V. Berinde, Iterative Approximation of Fixed Points, Springer, Berlin, Germany, 2007.

[31] R. Chugh and V. Kumar, "Data dependence of Noor and SP iterative schemes when dealing with quasi-contractive operators," International Journal of Computer Applications, vol. 40, no. 15, pp. 41-46, 2011. 


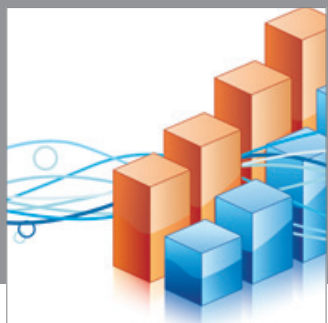

Advances in

Operations Research

mansans

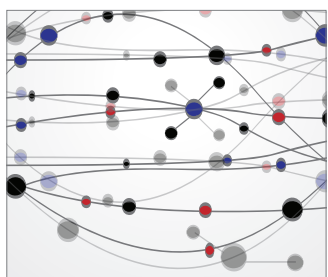

The Scientific World Journal
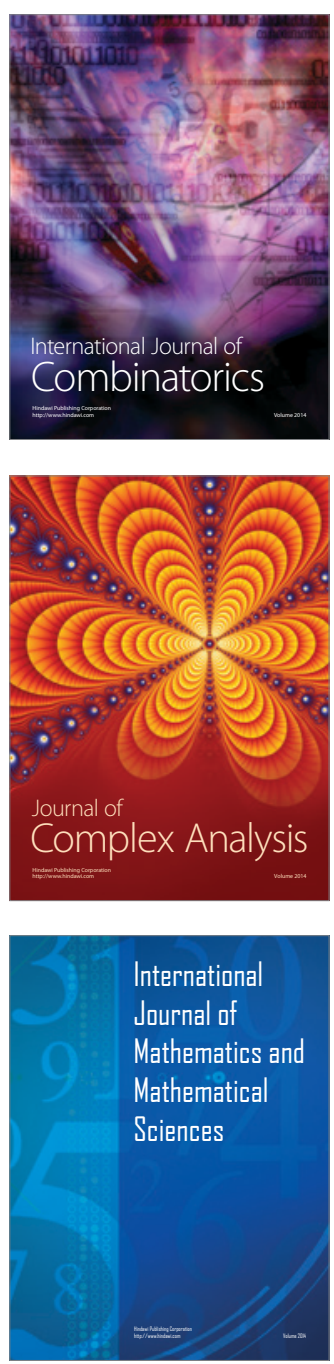
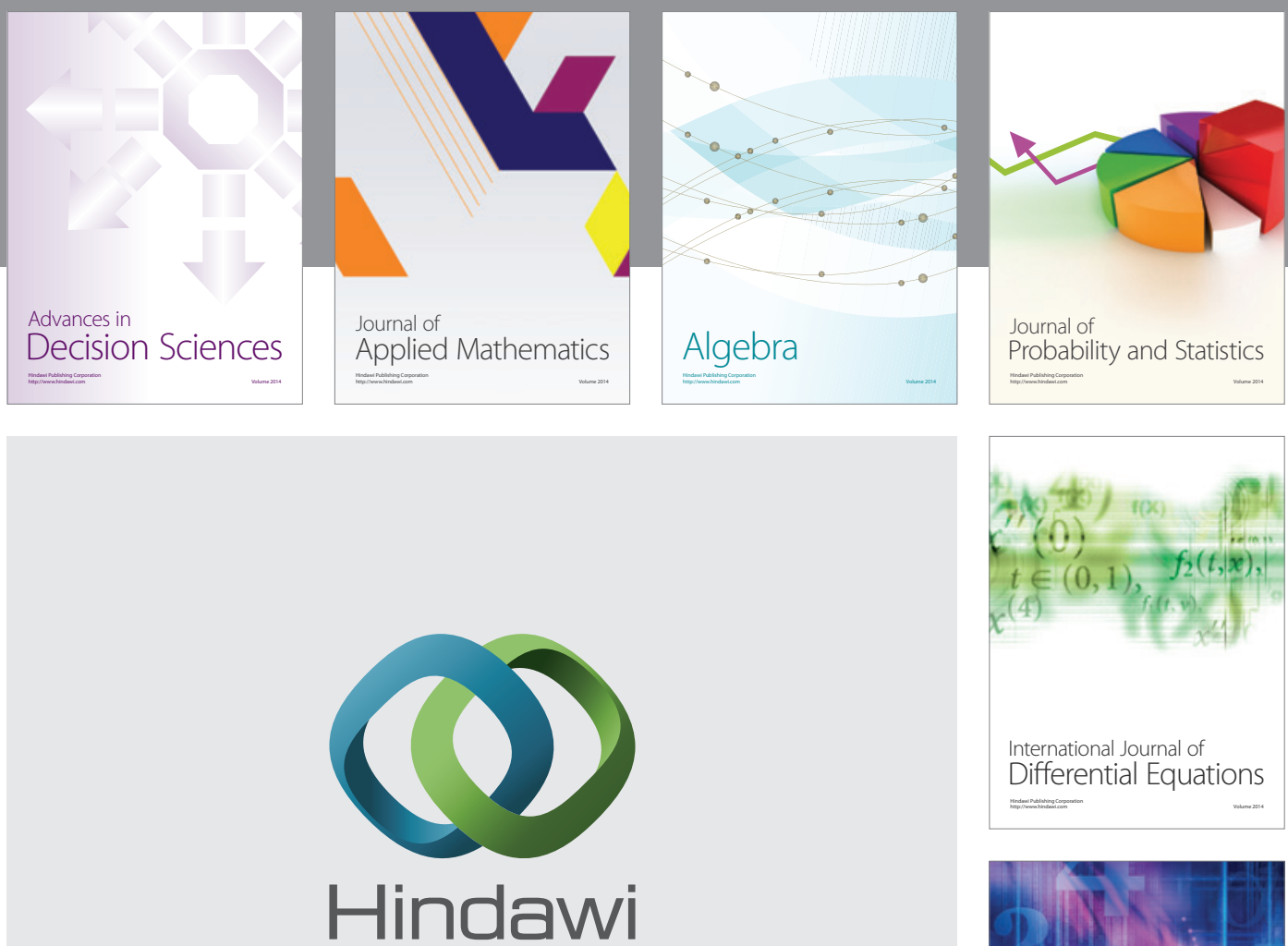

Submit your manuscripts at http://www.hindawi.com
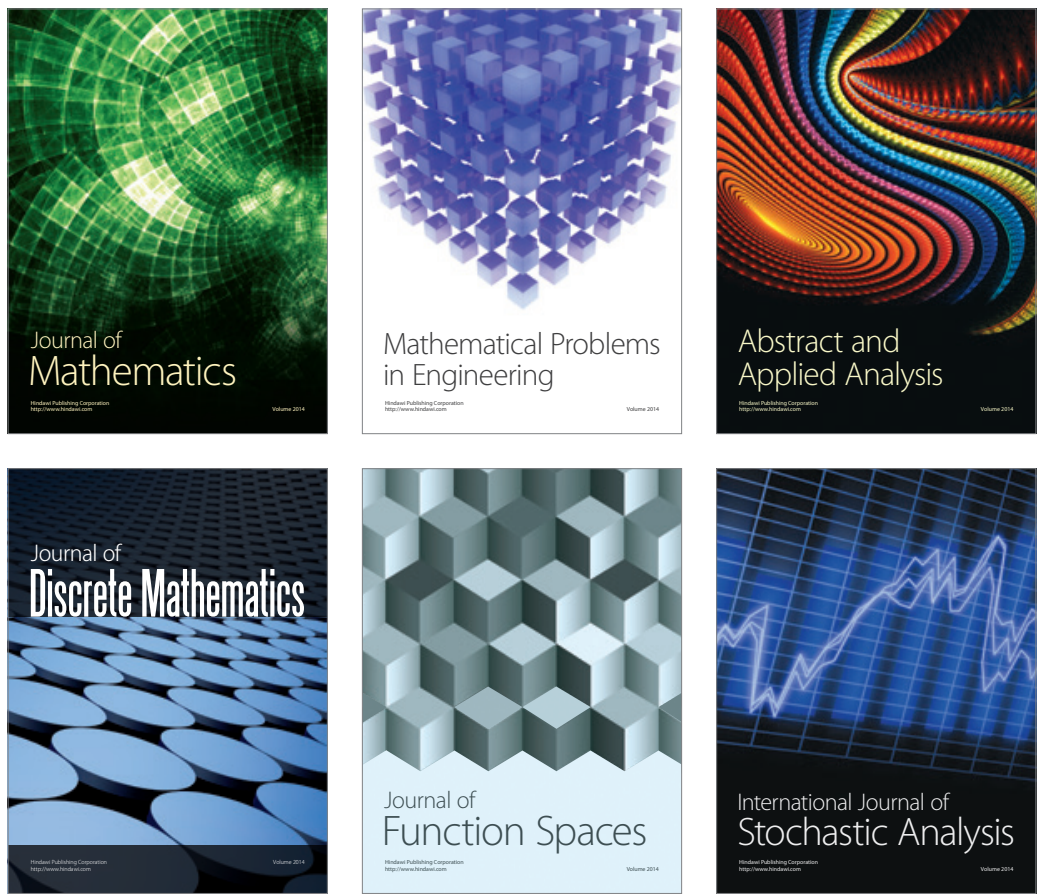

Journal of

Function Spaces

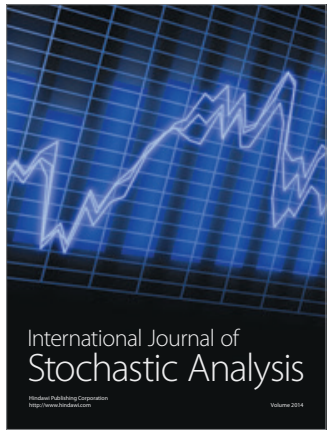

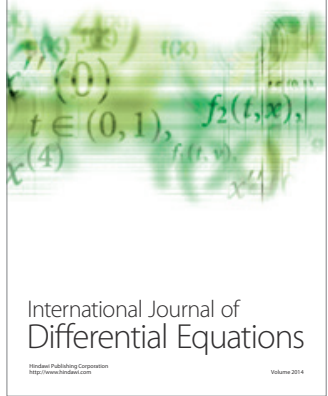
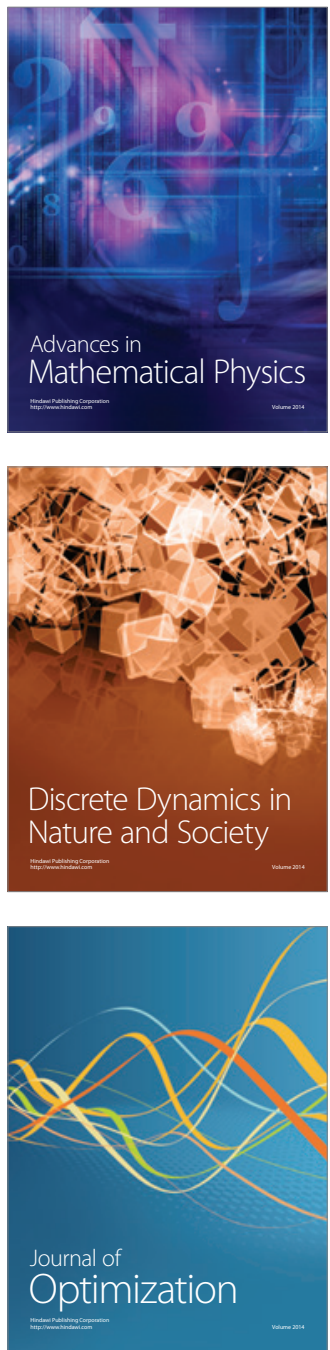